\title{
Tidal Tails around the Outer Halo Globular Clusters Eridanus and Palomar 15
}

\author{
G. C. Myeong ${ }^{1,2}$, Helmut Jerjen ${ }^{1}$, Dougal Mackey ${ }^{1}$, and Gary S. Da Costa ${ }^{1}$ \\ ${ }^{1}$ Research School of Astronomy and Astrophysics, The Australian National University, Canberra, ACT 2611, Australia \\ ${ }^{2}$ Institute of Astronomy, University of Cambridge, Madingley Road, Cambridge CB3 OHA, UK \\ Received 2017 March 13; revised 2017 April 24; accepted 2017 April 25; published 2017 May 11
}

\begin{abstract}
We report the discovery of tidal tails around the two outer halo globular clusters, Eridanus and Palomar 15, based on $g i$-band images obtained with DECam at the CTIO $4 \mathrm{~m}$ Blanco Telescope. The tidal tails are among the most remote stellar streams currently known in the Milky Way halo. Cluster members have been determined from the color-magnitude diagrams and used to establish the radial density profiles, which show, in both cases, a strong departure in the outer regions from the best-fit King profile. Spatial density maps reveal tidal tails stretching out on opposite sides of both clusters, extending over a length of $\sim 760 \mathrm{pc}$ for Eridanus and $\sim 1160 \mathrm{pc}$ for Palomar 15 . The great circle projected from the Palomar 15 tidal tails encompasses the Galactic Center, while that for Eridanus passes close to four dwarf satellite galaxies, one of which (Sculptor) is at a comparable distance to that of Eridanus.
\end{abstract}

Key words: globular clusters: general - globular clusters: individual (Eridanus, Palomar 15)

\section{Introduction}

It is widely accepted that large galaxies such as the Milky Way formed through accretion and merger of numerous protogalactic fragments (e.g., Searle \& Zinn 1978; Blumenthal et al. 1984). A significant fraction of the Milky Way's globular cluster population is thought to have been acquired by this process, and it is believed that a large portion of the current halo stellar mass may have been donated by their host dwarf galaxies via tidal disruption and mass loss (Mackey \& Gilmore 2004; Forbes \& Bridges 2010).

There are two types of stellar structures that we expect to see around Galactic globular clusters. One can occur around accreted clusters and represents the remnant of the disrupted parent dwarf galaxy (see Olszewski et al. 2009 and Kuzma et al. 2016). In extreme cases, we might see a cluster embedded in an extended stellar stream. This is the case in the halo of M31 (e.g., Mackey et al. 2010) and in our own Galaxy where a number of globular clusters are associated with the disrupting Sagittarius dwarf galaxy (e.g., Law \& Majewski 2010).

The second type consists of narrow stellar streams arising from the dynamical evolution of the cluster itself in the external tidal field of the Milky Way. Many studies have investigated the presence of tidal tails associated with globular clusters (e.g., Grillmair et al. 1995; Leon et al. 2000). It has been found that some globular clusters have stellar distributions that significantly differ from a King profile (King 1962), extending beyond the nominal tidal radius (Grillmair et al. 1995; Carballo-Bello et al. 2012).

Recently, it has been discovered that the tidal tails of some globular clusters, such as Palomar 5 (Odenkirchen et al. 2001; Grillmair \& Dionatos 2006) and NGC 5466 (Belokurov et al. 2006a; Grillmair \& Johnson 2006), extend over several hundred parsecs in physical length. In this context, it is also interesting to note that many narrow streams, like the Orphan (Belokurov et al. 2006b; Grillmair 2006; Grillmair et al. 2015) and Phoenix streams (Balbinot et al. 2016), exist in the inner Milky Way halo. These likely originate from completely disrupted globular clusters (Newberg et al. 2010; Martin et al. 2014).
The main point is that the Galactic tidal field, disk and bulge shocks, and two-body relaxation can all affect the outer structures of globular clusters in ways that we do not fully understand. Adding new examples of globular clusters with tidal tails, particularly at large Galactocentric distances, thus gives us additional information about the frequency of this phenomenon and can help to probe the outermost parts of the Galaxy. The properties of such streams can further help to constrain the dark matter distribution in the Galactic halo. For example, the gaps in the tidal tails of Palomar 5 may tell us about the dark matter sub-halos predicted in $\Lambda \mathrm{CDM}$ cosmology (Ngan \& Carlberg 2014).

So far, most studies searching for tidal structures around Milky Way globular clusters have been restricted to relatively nearby targets (e.g., Grillmair \& Carlin 2016) with the one exception of Palomar 14 (Sollima et al. 2011). In this Letter, we report the discovery of stellar substructures around two distant halo globular clusters, Eridanus $\left(R_{\mathrm{GC}}=95.0 \mathrm{kpc}\right)$ and Palomar $15\left(\mathrm{Pal} 15 ; R_{\mathrm{GC}}=38.4 \mathrm{kpc}\right)$.

\section{Data Analysis}

We used the Dark Energy Camera (DECam) at the CTIO $4 \mathrm{~m}$ Blanco Telescope to obtain deep imaging around Eridanus and Pal 15. This instrument comprises a 62 CCD mosaic that spans a $3 \mathrm{deg}^{2}$ field of view and has a pixel scale of 0 .' 27 (Flaugher et al. 2015). For Eridanus, we obtained 5 dithered $900 \mathrm{~s} g$-band images and 11 dithered $600 \mathrm{~s} i$-band images on 2014 February 25-27 (average seeing $\sim 1$ !' 11). For Pal 15, we obtained five dithered $360 \mathrm{~s} g$-band images and five dithered $360 \mathrm{~s} i$-band images on 2013 September 24-26 (average seeing 1". 12). The nights were part of the observing programs 2014A-0621 for Eridanus and 2013B-0617 for Pal 15-PI: D. Mackey. The raw images were preprocessed with the DECam Community Pipeline (Valdes et al. 2014), including application of the astrometric solution. We used the resampled images (individual frames) and their corresponding weight maps for our study.

SExtractor (Bertin \& Arnouts 1996) and PSFEx (Bertin 2011) were employed for source detection and PSF photometry. Star/galaxy separation was performed using the method described in Koposov et al. (2015), which adopts 

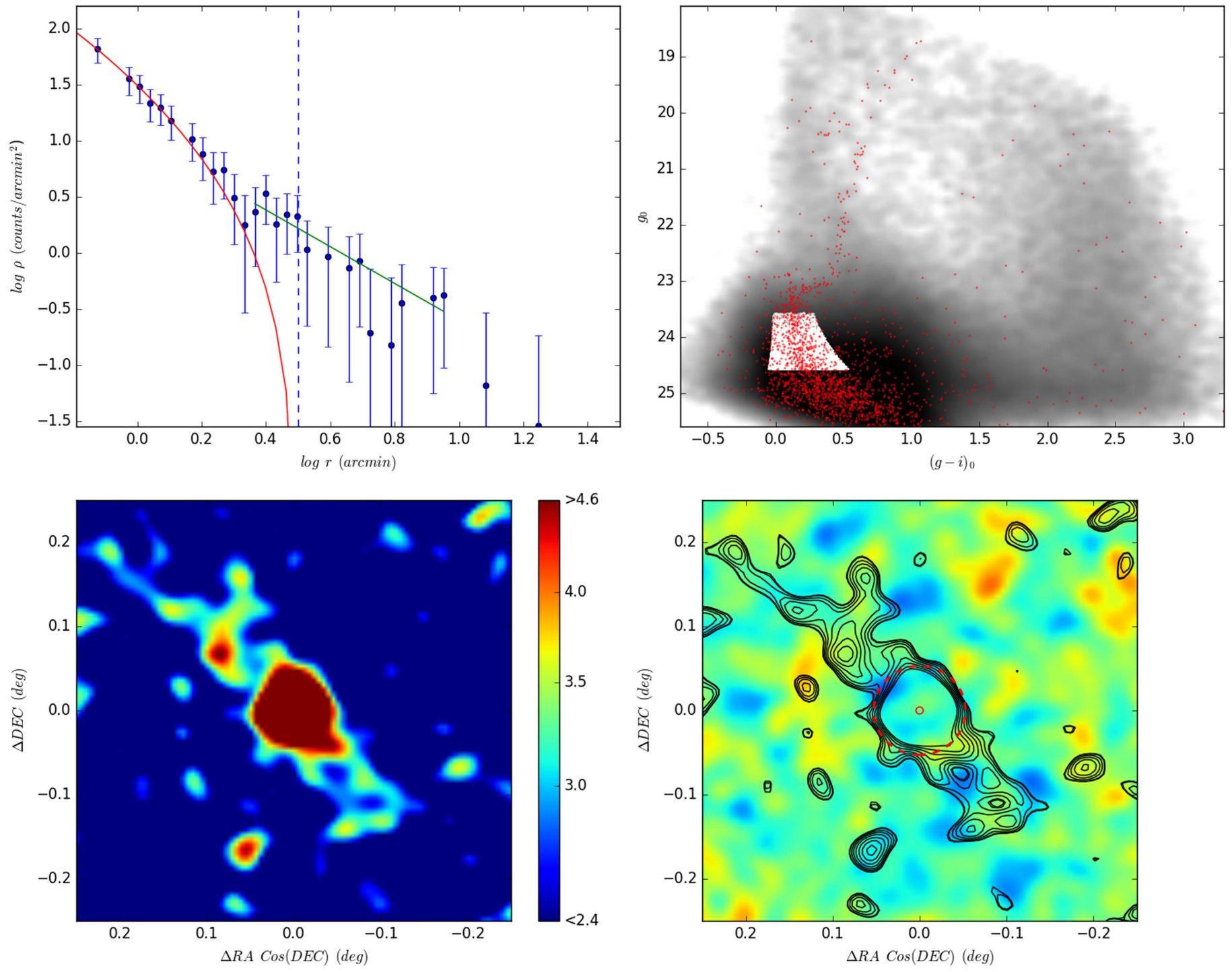

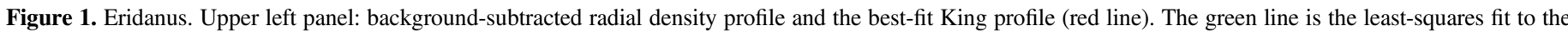

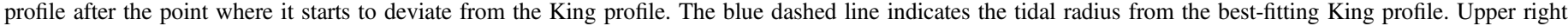

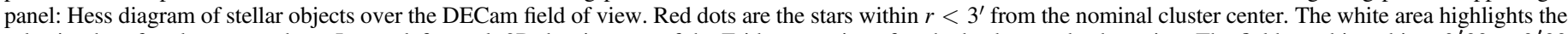

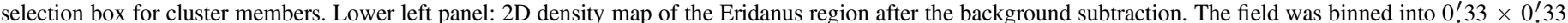

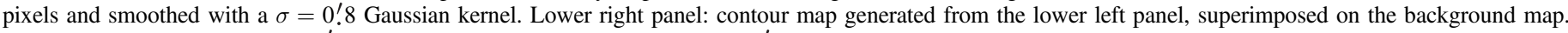

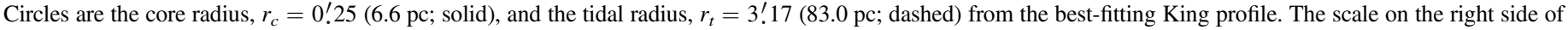
the density map is the signal strength in units of the standard deviation above the background. The contour lines range from $2.7 \sigma$ to $4.75 \sigma$ in logarithmic steps.

$\mid$ SPREAD_MODEL $\mid<$ SPREADERR_MODEL + 0.003 as the threshold for stars. The instrumental magnitudes were calibrated by cross-matching with the Pan-STARRS1 StackObjectThin catalog (Chambers et al. 2016) and then de-reddened using the Schlegel et al. (1998) dust maps with the extinction coefficients from Schlafly \& Finkbeiner (2011). The inferred distances are consistent with those given in Harris (1996).

For a given target we constructed the $\left((g-i)_{0}, g_{0}\right)$ colormagnitude diagram (CMD) using all stellar objects within $r<3^{\prime}$ from the nominal cluster center and fit a fiducial line representing the locus of cluster members in the CMD. The fiducial line for each cluster was determined empirically. The CMDs for the inner $3^{\prime}$, which are dominated by cluster members, were subdivided into intervals of $g_{0}$ and the $3 \sigma$ clipped median $(g-i)_{0}$ colors were determined. A continuous curve through the pairs of mean $g_{0}$ and the corresponding median color for each interval then define the fiducial line for the cluster members. This fiducial line was then used to calculate a weight $w$ for each star detected across the field. The weight quantifies the likelihood of a star being a cluster member based on its distance from the fiducial line along the color axis. The weight value was calculated using the Gaussian distribution $N(x, \mu, \sigma)$ centered on the fiducial line (normalized to have $w=1.0$ at the center), with $\sigma$ corresponding to the uncertainty in $(g-i)_{0}$ at the $g_{0}$ value, which were taken from the photometric errors generated by SExtractor.

Stars within a $2 \sigma$ range from the fiducial line in color (that is, $\left.w=N\left((g-i)_{0, *},(g-i)_{0, \text { fid }}, \sigma_{(g-i)_{0}}\right)>0.135\right)$ were considered to have a high probability of being related to the target cluster. We will henceforth call this selection "member stars" although it still contains some contamination from foreground/ background objects (henceforth "background") that happen to lie near the cluster population in CMD. To minimize this contamination while maximizing the signal due to cluster 

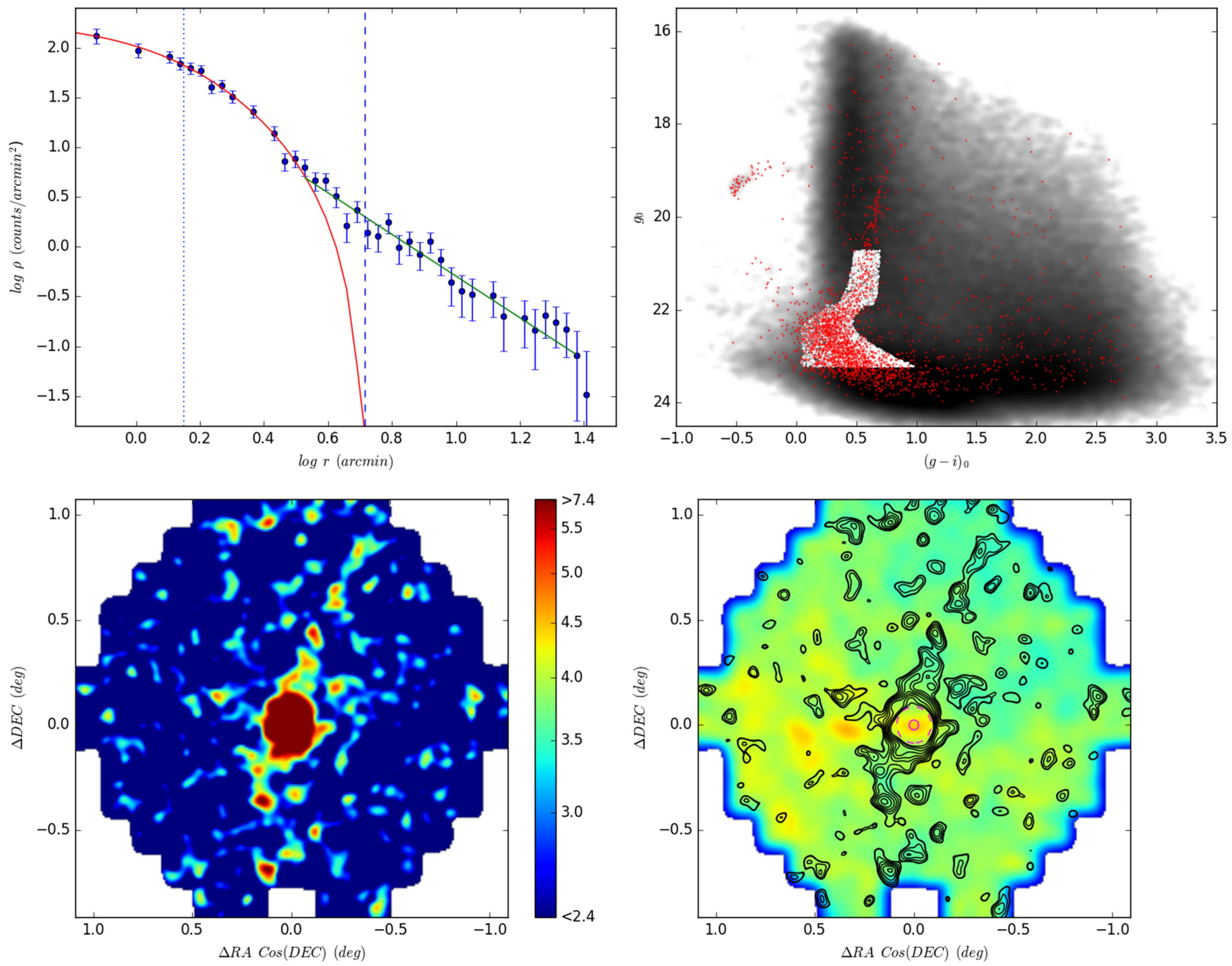

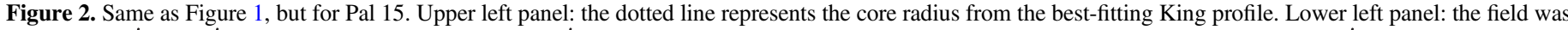

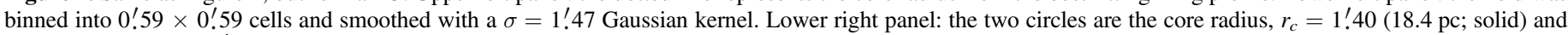
the tidal radius, $r_{t}=5$ !.19 (68.1 pc; dashed). The contour lines range from $3.0 \sigma$ to $8.85 \sigma$ in logarithmic steps.

members, we further restricted our selection to an interval in $g_{0}$ defined to cover the region on the CMD with the greatest contrast between "cluster" and "background" stars. In addition, we ensured that the faint end of this interval was 0.5 mag above the $50 \%$ photometric completeness limit.

Radial density profiles and spatial density maps were generated to investigate the distribution of the member stars (Figures 1 and 2). For the radial profile (upper left panel), we binned our field of view in concentric annuli with logarithmic spacing. Poisson statistics were assumed for calculating the uncertainty in each bin. The best-fitting King profile (King 1962) was determined by using a python library LIMFIT (Newville et al. 2014) after subtracting the background level. The background level was fixed from the total (cluster + background) density profile by determining the median annular density after $3 \sigma$-clipping.

Two-dimensional density maps (bottom panels) were constructed by binning the DECam field into cells of size 0 .33 $\times 0.33$ for Eridanus and $0.59 \times 0.59$ for Pal 15 , and then smoothing with a Gaussian kernel of width $\sigma=0$ '.8 for Eridanus and $\sigma=1 ! 47$ for Pal 15. The bin size and sigma were chosen after testing various combinations to provide the maximum contrast for the tidal features. The median stellar density of the field (=background), beyond 3 tidal radii from the cluster, is $5 \mathrm{star} \operatorname{arcmin}^{-2}\left(\sigma=2.9 \mathrm{star}_{\operatorname{arcmin}}^{-2}\right)$ for Eridanus and $7 \mathrm{star}_{\operatorname{arcmin}}^{-2} \quad\left(\sigma=3.1 \mathrm{star}_{\operatorname{arcmin}}^{-2}\right)$ for Pal 15 before smoothing. We generated a background map for each cluster in the same manner by using the corresponding subsample of background objects. After this map was normalized to the median $3 \sigma$-clipped density of the $w>0.135$ map determined above, it was subtracted to remove any large-scale gradients or instrumental artifacts across the field. Local rms values were measured for each of the $62 \mathrm{CCDs}$. The median was considered to be the background rms and used to scale the background-subtracted spatial density map (bottom left panel).

\section{Eridanus}

Eridanus, at $R_{\mathrm{GC}}=95.0 \mathrm{kpc}$ (Harris 1996), is one of the most distant Galactic globular clusters known. It has been classified as a "young" halo cluster, suggesting that it may have originated in an external satellite galaxy and been accreted into the Galactic halo 
Table 1

Basic Parameters for the Tidal Structures of Eridanus and Pal 15

\begin{tabular}{|c|c|c|c|c|c|c|}
\hline ID & $\begin{array}{c}r_{c} \\
(\mathrm{pc})\end{array}$ & $\begin{array}{c}r_{t} \\
(\mathrm{pc})\end{array}$ & $c$ & $\begin{array}{l}\operatorname{size}_{t 1} \\
(\mathrm{pc})\end{array}$ & $\begin{array}{l}\operatorname{size}_{\mathrm{t} 2} \\
(\mathrm{pc})\end{array}$ & $\begin{array}{c}\text { angle }_{\text {inc }} \\
\text { (deg) }\end{array}$ \\
\hline Eridanus & $6.6 \pm 3.1$ & $83.0 \pm 20.0$ & $1.10 \pm 0.23$ & $\sim 480$ & $\sim 280$ & $\sim 180$ \\
\hline Pal 15 & $18.4 \pm 2.0$ & $68.1 \pm 14.7$ & $0.57 \pm 0.11$ & $\sim 780$ & $\sim 380$ & $\sim 170$ \\
\hline
\end{tabular}

Note. $\left({ }_{\mathrm{t} 1}\right)$ indicates Tail $1,\left(_{\mathrm{t} 2}\right)$ indicates Tail 2, and (angle $\left.\mathrm{inc}_{\mathrm{c}}\right)$ indicates the angle between two tails.

(Zinn 1993; Mackey \& van den Bergh 2005). Our best-fitting King profile has a core radius $r_{c}=0{ }^{\prime} .25 \pm 0$. $12(6.6 \pm 3.1 \mathrm{pc})$ and a tidal radius $r_{t}=3 ! 17 \pm 0 ! 76(83.0 \pm 20.0 \mathrm{pc})$, with the concentration index $c=\log \left(r_{t} / r_{c}\right)=1.10 \pm 0.23$. These values agree well with previous measurements $\left(r_{c}=0.25\right.$ and $r_{t}=3$ !.15; Harris 1996). The radial profile exhibits an excess of stars outside $r \sim 1$ '.82 $(\log (r) \sim 0.26)$, which continues beyond the nominal tidal radius $r>r_{t}=3$ ! 17 and follows a power law with an index of $\gamma=-1.64 \pm 0.16$ (azimuthally averaged).

The spatial density map and the corresponding contour map (lower panels of Figure 1) reveal two prominent tidal tails extending considerably beyond the tidal radius (dashed circle in the contour map). These structures show no correlation with the background map. Tail 1 extends $\sim 18^{\prime}(\sim 480 \mathrm{pc})$ from the cluster center in the north-east direction at a position angle of $\mathrm{PA}=40^{\circ} \pm 5^{\circ}$. Tail 2 extends $\sim 11^{\prime}(\sim 280 \mathrm{pc})$ in the southwest direction $\left(\mathrm{PA}=220^{\circ} \pm 10^{\circ}\right)$. The fractional overdensity of cluster stars is $29.4 \%$ and $31.6 \%$, respectively, in the distance interval $2.5 r_{t}<r<3 r_{t}$. The alignment of the features is close to axisymmetric, suggesting that they are likely following the orbit of Eridanus. Given the alignment and the narrow width compared to the size of the cluster, it would seem likely that the features are tidal tails resulting from the dynamical evolution of Eridanus in the tidal field of the Milky Way.

\section{Palomar 15}

Pal 15 is an outer halo globular cluster at $R_{\mathrm{GC}}=38.4 \mathrm{kpc}$ (Harris 1996). Although it is classified as an "old" halo cluster, Pal 15 is a good accretion candidate by virtue of its location at a large Galactocentric distance (Mackey \& Gilmore 2004). Our best-fitting King profile has a core radius $r_{c}=1 ! 40 \pm$ 0 ! $15(18.4 \pm 2.0 \mathrm{pc})$ and a tidal radius $r_{t}=5 ! 19 \pm 1 ! 12$ $(68.1 \pm 14.7 \mathrm{pc})$, with the concentration index $c=\log \left(r_{t} / r_{c}\right)=$ $0.57 \pm 0.11$. This shows good agreement with previous measurements as listed in Harris $\left(1996 ; r_{c}=1\right.$ ! $\left.20, r_{t}=4 ! 77\right)$. The small concentration index of 0.57 , mainly a consequence of the exceptionally large core radius, suggests that $\mathrm{Pal} 15$ has been severely affected by the Galactic tidal field. Both Pal 5 and Pal 14 also have large core radii and are known to have extensive tidal tails (Odenkirchen et al. 2001; Sollima et al. 2011).

The radial profile of Pal 15 follows closely a King profile until the local star density has dropped to $\sim 3 \%$ of its central value $(r \sim 3 ! 47$ or $\log (r) \sim 0.54)$, beyond which there is a strong excess of stars that continues past the nominal tidal radius and follows a power law with an index of $\gamma=-2.09 \pm 0.09$ (azimuthally averaged).

The spatial density and contour maps (lower panels in Figure 2) show clear tidal tail features extending beyond the cluster's tidal radius (dashed circle in the contour map), crossing the field from the north-west to the south. These structures show no correlation with the background trend, nor with the extinction map (Schlegel et al. 1998; Schlafly \&
Finkbeiner 2011). Tail 1 extends $\sim 59^{\prime}$ ( $\sim 780 \mathrm{pc}$ ) from the cluster center in the north-west direction at a position angle of $\mathrm{PA}=340^{\circ} \pm 5^{\circ}$. At the $\sim 3.0 \sigma$ significance level it consists of two main fragments (see the contour map). We tested various combinations of binning sizes and smoothing kernels. The secondary, more distant fragment is visible in every solution. This supports the notion that this continuation of Tail 1 is indeed a real feature. The gap between the two segments may be similar to the gaps seen in the Pal 5 tails, whose origin has been the subject of a number of studies (e.g., Carlberg et al. 2012). Tail 2 extends $\sim 29^{\prime}(\sim 380 \mathrm{pc})$ in the south-east direction with a possible kink at half this length. The mean position angle is $\mathrm{PA}=150^{\circ} \pm 10^{\circ}$. The fractional overdensity of cluster stars is $20.9 \%$ and $22.5 \%$, respectively, in the distance interval $2.5 r_{t}<r<3 r_{t}$. Both tails have considerable sizes when compared to Pal 15's nominal tidal radius $\left(r_{t}=5\right.$ ! 19). Although the two tails are different in length, their alignment close to axisymmetric suggests that they are following the orbit of Pal 15. Additionally, both tidal tails are relatively narrow compared to the cluster size, indicating that these stellar streams are, like in the case of Eridanus, the result of the dynamical evolution in the Galactic tidal field.

\section{Discussion}

We have discovered tidal tails extending from the two remote Milky Way globular clusters Eridanus and Pal 15. The narrowness of the tails compared to the cluster size, along with their symmetric orientation on either side of the cluster centers indicates that they are due to the loss of cluster members to the Galactic tidal field, as seen for several other globular clusters such as Pal 5 and NGC 5466 and in numerical simulations (Combes et al. 1999; Capuzzo Dolcetta et al. 2005). Moreover, the power-law slope for each tail region $\left(\mathrm{PA} \pm 10^{\circ}\right)$ shows $\gamma=-1.20 \pm 0.19$ and $\gamma=-1.25 \pm 0.16$ for Eridanus, $\gamma=-1.24 \pm 0.12$ and $\gamma=-1.26 \pm 0.12$ for Pal 15, which are similar values as in the case of Pal 5 (Odenkirchen et al. 2003) and steeper than constant density.

Table 1 contains the estimated basic parameters for the tidal tails. Both pairs exhibit significant extent beyond the nominal tidal radius of their respective cluster-the tails of Eridanus span $\sim 760$ pc in total, while those for Pal 15 trace $\sim 1160$ pc.

The tails of Pal 15 may well extend beyond the edge of the DECam field of view. In this case, our study provides a lower limit on their length, and some additional off-field data will be required to test the true extent of Pal 15's tidal structure. In addition, the tails appear curved, especially if the fragment near the southern edge of our Pal 15 field is considered to be part of the structure. If confirmed, this could hold information about the underlying Galactic potential or it may be simply related to projection effects (Combes et al. 1999).

For Eridanus, the tidal tails are well confined to the inner part of our DECam field. This suggests that we have effectively found their full extent, unless the star densities in the tails are 


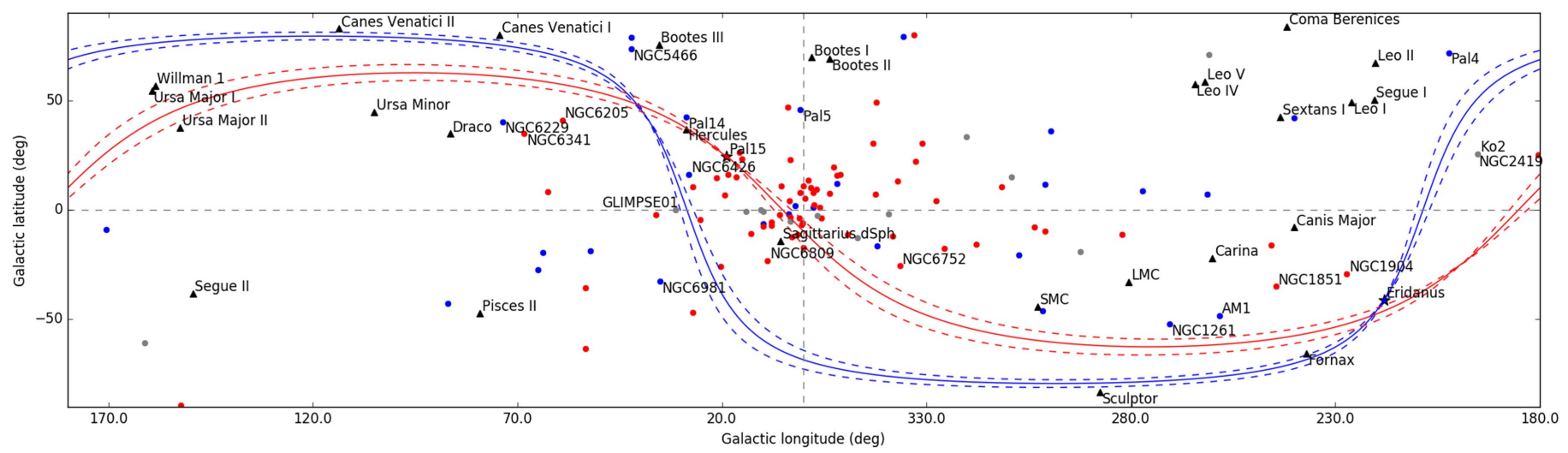

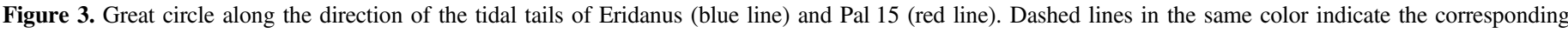

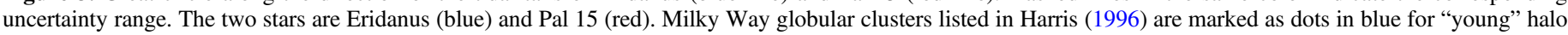

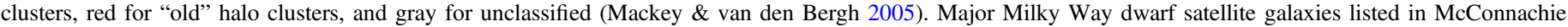
(2012) are marked with black triangles. Vertical and horizontal gray dashed lines indicate $0^{\circ}$ of Galactic longitude and latitude, respectively.

getting too low relative to the background. This may indicate an eccentric orbit of Eridanus about the Milky Way as we may be seeing its long tails foreshortened.

We generated the great circle along the direction of the tidal tails for each cluster, based on the position angle and its uncertainty estimated for each tail (Figure 3). We compared its path with the positions of the Milky Way globular clusters listed in Harris (1996) and the Milky Way dwarf satellites in McConnachie (2012). Since the tidal tails are expected to be projected along the orbital path of the cluster (Combes et al. 1999; Capuzzo Dolcetta et al. 2005), this great circle can provide a rough estimate for the cluster's orbit. It is notable that the potential orbit of Eridanus passes close to the dwarf galaxies Canes Venatici I, Canes Venatici II, Fornax, and Sculptor. Sculptor $\left(R_{\mathrm{GC}}=86 \mathrm{kpc}\right.$; McConnachie 2012) has a comparable distance to that of Eridanus. Since Eridanus is classified as a "young" halo cluster based on its metallicity and horizontal branch morphology (Mackey \& van den Bergh 2005), and indeed exhibits a CMD consistent with an age up to $\sim 2 \mathrm{Gyr}$ younger than the oldest Milky Way globular clusters (Stetson et al. 1999), this possible association to Sculptor and/or another of the three satellites might provide additional evidence of Eridanus's extragalactic origin (see also Keller et al. 2012). Even so, it is striking that despite several indications that both Eridanus and Pal 15 might be accreted, and our data being sensitive enough to detect low surface brightness features at their distances, no clear evidence for stellar debris from parent dwarf galaxies was observed. Carballo-Bello et al. (2015) suggested that Eridanus may be associated with the Monoceros ring based on the modeled orbit of this structure by Penarrubia et al. (2005); however, the great circle of Eridanus disagrees with this modeled orbit, suggesting no clear association between them.

The great circle defined by the tidal tails of Pal 15 passes close to Ursa Major II, which also has a comparable distance of $R_{\mathrm{GC}}=38 \mathrm{kpc} \quad$ (McConnachie 2012), suggesting possible association. We further notice that the potential orbits of Eridanus and Pal 15 extend to relatively high Galactic latitudes, indicating that the clusters may be on plunging orbits relative to the Galactic disk. Assuming the orbits are sufficiently eccentric to pass through the inner region of the Milky Way, the clusters could experience periodic disk and/or bulge shocks. Since these processes are known to be destructive (Gnedin \& Ostriker 1997; Gnedin et al. 1999), this could explain why both clusters exhibit tidal tails. A prime example is Pal 5, which suffers this type of tidal shock and is predicted to be destroyed at its next disk crossing (Dehnen et al. 2004).

In the case of Eridanus $\left(R_{\mathrm{GC}}=95.0 \mathrm{kpc}\right)$, we have discovered a new tidal stream in an extremely remote part of the Galaxy which is poorly understood and where no other narrow streams are known. Studying Eridanus and its tails in detail may lead us to a better understanding of the gravitational potential in the extreme outskirts of the Galaxy. A key aspect of this will be understanding whether the tails arise from the action of the tidal field at this large Galactocentric distance, or whether Eridanus is on a very eccentric orbit such that it passes through the inner Milky Way, and the tails are more likely due to the action of the tidal field at smaller radii.

The authors acknowledge the support of the Australian Research Council through Discovery projects DP150100862 and DP150103294. We also thank the referee for comments and suggestions, which helped to improve the quality of the publication. G.C.M. thanks Boustany Foundation, Cambridge Trust, and Issac Newton Studentship for their support on his work. This project used data obtained with the Dark Energy Camera (DECam), which was constructed by the Dark Energy Survey (DES) collaboration. Funding for the DES Projects has been provided by the DOE and NSF (USA), MISE (Spain), STFC (UK), HEFCE (UK). NCSA (UIUC), KICP (U. Chicago), CCAPP (Ohio State), MIFPA (Texas A\&M), CNPQ, FAPERJ, FINEP (Brazil), MINECO (Spain), DFG (Germany), and the collaborating institutions in the Dark Energy Survey, which are Argonne Lab, UC Santa Cruz, University of Cambridge, CIEMAT-Madrid, University of Chicago, University College London, DES-Brazil Consortium, University of Edinburgh, ETH Zürich, Fermilab, University of Illinois, ICE (IEEC-CSIC), IFAE Barcelona, Lawrence Berkeley Lab, LMU München and the associated Excellence Cluster Universe, University of Michigan, NOAO, University of Nottingham, Ohio State University, University of Pennsylvania, University of Portsmouth, SLAC National Lab, Stanford University, University of Sussex, and Texas A\&M University.

\section{References}

Balbinot, E., Yanny, B., Li, T. S., et al. 2016, ApJ, 820, 58

Belokurov, V., Evans, N. W., Irwin, M. J., Hewett, P. C., \& Wilkinson, M. I. 2006a, ApJL, 637, L29

Belokurov, V., Zucker, D. B., Evans, N. W., et al. 2006b, ApJL, 642, L137 
Bertin, E. 2011, in ASP Conf. Ser. 442, Astronomical Data Analysis Software and Systems XX, ed. I. N. Evans et al. (San Francisco, CA: ASP), 435

Bertin, E., \& Arnouts, S. 1996, A\&AS, 117, 393

Blumenthal, G. R., Faber, S. M., Primack, J. R., \& Rees, M. J. 1984, Natur, 311,517

Capuzzo Dolcetta, R., Di Matteo, P., \& Miocchi, P. 2005, AJ, 129, 1906

Carballo-Bello, J. A., Gieles, M., Sollima, A., et al. 2012, MNRAS, 419, 14

Carballo-Bello, J. A., Muñoz, R. R., Carlin, J. L., et al. 2015, ApJ, 805, 51

Carlberg, R. G., Grillmair, C. J., \& Hetherington, N. 2012, ApJ, 760, 75

Chambers, K. C., Magnier, E. A., Metcalfe, N., et al. 2016, arXiv:1612.05560

Combes, F., Leon, S., \& Meylan, G. 1999, A\&A, 352, 149

Dehnen, W., Odenkirchen, M., Grebel, E. K., \& Rix, H.-W. 2004, AJ, 127, 2753

Flaugher, B., Diehl, H. T., Honscheid, K., et al. 2015, AJ, 150, 150

Forbes, D. A., \& Bridges, T. 2010, MNRAS, 404, 1203

Gnedin, O. Y., \& Ostriker, J. P. 1997, ApJ, 474, 223

Gnedin, O. Y., Lee, H. M., \& Ostriker, J. P. 1999, ApJ, 522, 935

Grillmair, C. J. 2006, ApJL, 645, L37

Grillmair, C. J., \& Carlin, J. L. 2016, in Astrophysics and Space Science

Library, Vol. 420, ed. H. J. Newberg \& J. L. Carlin (Berlin: Springer), 87

Grillmair, C. J., \& Dionatos, O. 2006, ApJL, 641, L37

Grillmair, C. J., \& Johnson, R. 2006, ApJL, 639, L17

Grillmair, C. J., Freeman, K. C., Irwin, M., \& Quinn, P. J. 1995, AJ, 109, 2553

Grillmair, C. J., Hetherington, L., Carlberg, R. G., \& Willman, B. 2015, ApJL, 812, L26

Harris, W. E. 1996, AJ, 122, 1487

Keller, S. C., Mackey, D., \& Da Costa, G. S. 2012, ApJ, 744, 57

King, I. R. 1962, AJ, 67, 471

Koposov, S. E., Belokurov, V., Torrealba, G., \& Evans, N. W. 2015, ApJ, 805,130
Kuzma, P. B., Da Costa, G. S., Mackey, A. D., \& Roderick, T. A. 2016, MNRAS, 461, 3639

Law, D. R., \& Majewski, S. R. 2010, ApJ, 718, 1128

Leon, S., Meylan, G., \& Combes, F. 2000, A\&A, 359, 907

Mackey, A. D., \& Gilmore, G. F. 2004, MNRAS, 355, 504

Mackey, A. D., \& van den Bergh, S. 2005, MNRAS, 360, 631

Mackey, A. D., Huxor, A. P., Ferguson, A. M. N., et al. 2010, ApJL, 717, L11

Martin, N. F., Ibata, R. A., Rich, R. M., et al. 2014, ApJ, 787, 19

McConnachie, A. W. 2012, AJ, 144, 4

Newberg, H. J., Willett, B. A., Yanny, B., \& Xu, Y. 2010, ApJ, 711, 32

Newville, M., Stensitzki, T., Allen, D. B., \& Ingargiola, A. 2014, LMFIT: Nonlinear Least-square Minimization and Curve-fitting for Python, Zenodo, doi:10.5281/zenodo.11813

Ngan, W. H. W., \& Carlberg, R. G. 2014, ApJ, 788, 181

Odenkirchen, M., Grebel, E. K., Dehnen, W., et al. 2003, AJ, 126, 2385

Odenkirchen, M., Grebel, E. K., Rockosi, C. M., et al. 2001, ApJL, 548, L165

Olszewski, E. W., Saha, A., Knezek, P., et al. 2009, AJ, 138, 1570

Peñarrubia, J., Martínez-Delgado, D., Rix, H. W., et al. 2005, ApJ, 626, 128

Schlafly, E. F., \& Finkbeiner, D. P. 2011, ApJ, 737, 103

Schlegel, D. J., Finkbeiner, D. P., \& Davis, M. 1998, ApJ, 500, 525

Searle, L., \& Zinn, R. 1978, ApJ, 225, 357

Sollima, A., Martínez-Delgado, D., Valls-Gabaud, D., \& Peñarrubia, J. 2011, ApJ, 726, 47

Stetson, P. B., Bolte, M., Harris, W. E., et al. 1999, AJ, 117, 247

Valdes, F., Gruendl, R. \& DES Project 2014, in ASP Conf. Ser. 485, Astronomical Data Analysis Software and Systems XXIII, ed. N. Manset \& P. Forshay (San Francisco, CA: ASP), 379

Zinn, R. 1993, in ASP Conf. Ser. 48, The Globular Cluster-Galaxy Connection, ed. G. H. Smith \& J. P. Brodie (San Francisco, CA: ASP), 38 